\title{
Understanding Dyslexia: A Review of Contemporary Neuropsychological Theories and Cognitive-Behavioral Evidence
}

Rita Barakat

Neuroscience Graduate Program (NGP), University of Southern California

\begin{abstract}
Dyslexia is one of the most common reading disorders observed in children and adults across the world, yet it is also one of the most challenging for clinicians to diagnose and treat. The obstacles associated with detecting dyslexia and intervening with appropriate remediation therapies early in development largely stem from the heterogeneity of symptom presentation, complicated further by the delayed timeline for acquiring literacy. Over the last several decades and until recently, neuropsychologists and linguists alike have posited numerous theories and models to aid in understanding the underlying causes of dyslexia. The following review presents a selection of the leading theories for explaining dyslexia from the behavioral literature, as well as the cognitive-behavioral evidence that supports these theories. In addition, a brief exploration of the genetic factors influencing dyslexia is covered, as well as a number of key findings from neuroimaging research that demonstrate specific neuroanatomical and functional perturbations which may serve as predictors of dyslexia in pre-literate children.
\end{abstract}

Key Terms: Dyslexia, developmental dyslexia, reading disability, neuropsychology of reading 


\section{Introduction}

Dyslexia is a disorder characterized by difficulties in reading, specifically related to the ability to accurately and efficiently recognize words (Peterson and Pennington, 2012). While the ultimate purpose of reading is to comprehend what is being read, dyslexia is a disorder of decoding individual words, leaving overall comprehension intact. Previous clinical definitions of dyslexia have conflated the disorder with a more global "reading disability", but this is in fact an inaccurate representation of deficits unique to dyslexia, which are often distinctly different from those of other reading disorders (Shaywitz and Shaywitz, 2005). In addition to confusion relating to the comprehension versus decoding aspects of reading, there have also been many misrepresentations of dyslexia as being a disorder associated with low IQ. Recent studies of individuals with dyslexia have shown that this is not always necessarily the case, and in fact, there are many instances in which dyslexia exists in conjunction with high IQ, leading researchers to question whether in fact diagnostic measures of dyslexia should be related to IQ measures (Peterson and Pennington, 2012).

Dyslexia has been studied extensively across many disciplines and cultural groups, and the etiology remains consistent despite morphological and phonological differences between global languages. More specifically, developmental dyslexia refers to dyslexia that is detected in an individual or population in which literacy maturation has not yet been reached, yet pronounced difficulties in word recognition are evident throughout the course of acquiring literacy (Shaywitz and Shaywitz, 2005). Currently, there are no "one-size-fits-all" approaches for diagnosing developmental dyslexia, which can ultimately lead to educational delays in children who present the symptoms of dyslexia, but remain undiagnosed and are thus not treated early on their literacy training. In addition, many studies have demonstrated several psychological and neurobiological phenomena associated with dyslexia, however, few have yet to be isolated as a particular causal candidate of the disorder.

Several notable researchers in the fields of neuropsychology and cognitive psychology have presented compelling behavioral and cognitive evidence to support theories of the underlying cause(s) of dyslexia, with the ultimate goal of characterizing behavioral and biological observations as being either associated with/ co-morbid with dyslexia, or potentially causal of dyslexia. The following paragraphs will give attention to each of these theories and their supporting evidence, as well as the specific experimental designs that have been implemented to examine these theories.

\section{Phonological Deficit Theory}

Currently considered the reigning theory explaining the underlying causal components of dyslexia, the phonological deficit theory states that individuals with dyslexia have an impairment in the representation, storage and retrieval of the phonological aspects of language, or put more simply, the "speech sounds" (Ramus et al., 2003). Linguistic support for this theory stems from the fundamental process of acquiring literacy, which involves learning the association between graphemes and phonemes in the alphabetic system of a language: in other words, learning to associate individual letters with their corresponding sounds. The phonological deficit theory postulates that there is a deficit in the formation of these grapheme-phoneme associations, which ultimately impacts the ability to process the alphabetic system more globally (Ramus et al., 2003). Behavioral observations of individuals 
with dyslexia also provide strong support for this theory, as it remains largely undisputed that dyslexia is a disorder of phonological processing, though there are several competing theories explaining this particular deficit. Thus, the phonological deficit theory provides the most direct association between the underlying cognitive processes of reading and the behavioral manifestations observed in individuals with dyslexia, namely those pertaining to deficits in phonological awareness (Share and Stanovich, 1995).

There have also been several structural and functional neuroimaging studies that provide support for this theory, isolating left-hemisphere brain regions in the perisylvian cortex (temporal lobe areas immediately surrounding the sylvian fissure) as being implicated in phonological processing. However, the neuroimaging evidence supporting this theory is perhaps the most contested, as other studies using structural and functional magnetic resonance imaging (MRI/ fMRI) have identified more dispersed white matter tracts, some of which cross through these perisylvian regions, as well as more global networks pertaining to sensory and motor function, as being implicated in the phonological deficits observed in dyslexia (Ramus et al., 2003). Overall, the conflicting neurobiological evidence suggests that while the hallmark symptom of dyslexia may be difficulties in phonological processing, the underlying regions/ networks responsible for this deficit may not be isolated to those that are specifically implicated in phonological processing.

A comprehensive study by Ramus et al. examining several competing theories of dyslexia found particular support for the phonological deficit theory, when testing a population of seventeen university students with diagnosed dyslexia. The students underwent a battery of neuropsychological, phonological, auditory, visual and cerebellar tests to assess their cognitive abilities in each of these particular domains, each of which is tied to a theory of the underlying causality of dyslexia. The researchers found that a significant portion of their subjects performed below the standardized average for their age/ IQ/ educational level on the phonological tests, and the specific pattern of this low performance was homogeneous across the subject population (i.e. scores on each of the individual phonological tests were highly similar). This led the group to conclude that deficits in the phonological representation of words are "sufficient cause" of the observed behavioral manifestations of dyslexia within their particular subject population.

The researchers do note that several of the subjects also presented deficits in auditory processing, as evidenced from these subjects' low performance on the auditory behavioral tasks, however, they speculate that based on the heterogeneity of the auditory task scores, it is most likely not linked to the observed phonological deficits in a meaningful way. Another aspect of this study that is important to consider is the nature of the study design, which did not account for differences in reading level or educational background between the subject population used for the study and a more representative (random) pool of individuals with dyslexia, which the researchers noted was a weakness of the study design.

\section{Multiple Deficit Hypothesis}

In the mid- $21^{\text {st }}$ century, many additional behavioral disorders were increasingly observed as being comorbid with dyslexia (such as ADHD and Speech Sound Disorder, or SSD). Researchers attempted to explain these comorbidities by hypothesizing that the underlying causality of dyslexia may not be a single cognitive process, but rather, many 
cognitive processes that, when individually disrupted, result in the downstream etiology of dyslexia. This hypothesis is known as the Multiple Deficit Hypothesis, and was presented as a possible explanation for the myriad behavioral deficits observed in individuals with dyslexia by Bruce Pennington in 2006. Pennington argued that the framework for understanding the cognitive and behavioral components of dyslexia was fundamentally in contradiction with itself, as dyslexia is often characterized as a heterogeneous disorder in the context of the behavioral manifestations, but has traditionally been thought of as homogeneous in the context of the underlying cognitive processes affected (Pennington, 2006). In order to resolve this contradiction, Pennington and others have presented a "multiple cognitive deficit model" that explains the comorbidity of dyslexia and other behavioral/ attentional disorders, offering a contrast to the "single deficit" (also known as the phonological deficit) theory formalized by Ramus et al. in 2003.

Pennington and others went on to examine the cognitive, behavioral, clinical and statistical relationship between dyslexia and other disorders, primarily focusing on its comorbidity with ADHD, and these studies provide extensive evidence for the multiple deficit framework of dyslexia (Pennington, 2006). For example, in the original paper discussing this multiple deficit hypothesis, Pennington reviewed the hereditary evidence linking dyslexia and ADHD, to show that the overlap between these two disorders exists not in a diagnostic realm (the diagnostic tests that are used to assess these disorders do not overlap at all), but in a genetic realm. By studying monozygotic (MZ, or identical) and dizygotic (DZ, or fraternal) twins, one can assess the "bivariate heritability" of two disorders, which exists when the relationship between these disorders is stronger in MZ twins than DZ twins (Pennington, 2006). A meta-analysis of studies using $M Z$ and $D Z$ twins showed there is high bivariate heritability between dyslexia and ADHD, indicating that the underlying cognitive processes affected in both of these disorders may overlap more than previously expected.

In a more recent paper revisiting the multiple deficit hypothesis and other theories of dyslexia, Pennington's group performed extensive statistical analyses to test the relative clinical effectiveness of these models in diagnosing developmental dyslexia in pre-literate subject populations (Pennington et al. 2012). The results of these analyses were surprising in that the multiple deficit model did not in fact provide the greatest explanatory power for the deficits observed in children with dyslexia, leading Pennington to conclude that this and other models for understanding dyslexia should be considered as "probabilistic" predictors rather than "deterministic" frameworks for examining individuals with dyslexia. Pennington discusses the relative merits of the more contemporary "hybrid model" of dyslexia, and points out that various factors related to a child's family history (hereditary factors), age (important for considering reading level) and potential comorbidity with ADHD should be considered when diagnosing dyslexia.

\section{Double Deficit Hypothesis}

The double deficit hypothesis of dyslexia postulates that the behavioral outcomes observed in individuals with dyslexia are related to deficits in two individual, yet inextricably linked, word processing streams: the orthographic and the phonological streams. Maryanne Wolf and Patricia Greig Bowers were among the first to note that the difficulties in phonological processing in developmental dyslexia were also associated with slow symbol naming speed, 
leading them to argue that orthographic processing, critical in the visual assessment of words, should be considered in conjunction with phonological processing in the context of reading ability more broadly (Wolf and Bowers, 1999). Wolf and Bowers point out that naming speed has typically been considered a component of phonological processing, but it contributes to the significant variance in reading ability across individuals, independent of phonological processing, implying that the orthographic codes associated with words are necessary for fluent reading, in conjunction with phonological codes.

This theory returns to one of the central tenants presented in the phonological (single) deficit theory of dyslexia: that the grapheme-phoneme association between letters in the alphabet must be established early on in a child's literacy education, in order for the child to acquire reading fluency (Ramus et al., 2003). In re-examining this aspect of the phonological deficit theory, it is evident that in order for the correspondence between letters and sounds to be truly effective in establishing literacy, the downstream orthography of an entire word must be linked to the phonology of that word, as well as the individual morphemes that comprise the word.

Many behavioral studies provide support for the double deficit theory by demonstrating how problems with orthographic processing, when examined separately from phonological processing, result in lower reading performance than predicted for a given sample population. Manis et al. (1990) found that a significant sub-population of individuals with diagnosed reading deficits showed poorer orthographic processing skills than their reading recognition scores would have originally predicted. In addition, these individuals appeared to continuously rely on a phonological coding strategy that was not always effective in the absence of an orthographic memory for words. By examining these findings in the context of this double deficit theory, one can conclude that an attempt to compensate for a deficit in one of these word-coding strategies by relying on the other will still result in behavioral symptoms characteristic of dyslexia, as overall reading fluency requires both of these word coding strategies.

\section{Visual Attention Hypothesis}

In somewhat of a departure from the previous three theories of dyslexia, the visual attention (VA) hypothesis proposes that a limitation in the number of visual elements that can be processed in parallel within the visual field (also known as the visual attention span) result in the behavioral manifestations of dyslexia, independent of deficits in phonological awareness. Bosse et al. (2007) studied two independent populations of adults with dyslexia, one French and one British, and observed that the visual attention (VA) span accounted for reading performance in both groups, even after controlling for other factors such as IQ, verbal fluency, vocabulary and single letter identification skills. This led the group to conclude that deficits beyond phonological awareness not only contribute to the behavioral manifestations of dyslexia, could in fact be the core elements of the disorder.

Other neuropsychological evidence supporting the VA hypothesis comes from studies of the attentional blink $(A B)$, or the deficit in the ability to identify a second target following the first target, when both appear randomly within a sequence of other distractor items (foils). In a particular study conducted by Judy Buchholz and Anne Aimola Davies, the AB of five adults with dyslexia were compared to those of normal adult readers (Buchholz and Davies, 2007). 
They found that the $A B$ depends on the nature of the particular task administered, and this was true for both, the dyslexia and control subject groups. They also concluded that the attentional system more broadly is compromised in individuals with dyslexia, however, the authors did not go so far as to claim that this attentional deficit may be a core factor of dyslexia, but rather, one that may be associated with, or perhaps co-morbid with, dyslexia, and is thus important to consider in the context of reading ability.

\section{Amplitude Onset Deficit Hypothesis}

The amplitude onset deficit hypothesis is one that is primarily concerned with the early developmental (pre-literacy) processing of acoustic elements of speech and other auditory stimuli, which are universal across all languages (Goswami et al., 2011). In order for infants to begin to parse sounds as being specific to speech and prosody, auditory stimuli must be segmented into what these infants will later perceive to be words and sentences. Deficits in this early stage of processing auditory stimuli can lead to deficits in phonological processing once children are at the stage of acquiring literacy, by which point effective intervention may be too late. Usha Goswami and colleagues have argued in the last five years that a neurobiological marker for this deficit in auditory sensory processing is necessary in order to form an effective detection method for early developmental dyslexia, and that these fundamental sensory processing deficits are the root cause of the difficulties in phonological processing characteristic of dyslexia.

In a 2011 study, Goswami's group examined preliterate children from three language backgrounds: English, Spanish and Chinese. They used the rate of onset of the amplitude envelope (rise time) as the measure for effective parsing of sensory information into speech-related stimuli, as it is known that the rise time is critical in rhythmic processing of speech (Goswami et al., 2011). The results show that rise time sensitivity was a significant predictor of phonological processing ability, and was in fact the only significant predictor of reading acquisition, regardless of language. These findings lead the authors to postulate that early remediation therapies targeting the rhythm of speech sounds, as opposed to the phonological aspects of speech, may be more effective at treating the root cause of dyslexia preemptively.

\section{Assessing Family Risk Factors}

A large body of literature suggests that there is a strong relationship between family risk factors related to dyslexia and onset of the disorder during childhood. Logistically-speaking, the ability to assess various aspects of reading ability in relation to family history of dyslexia throughout the course of a child's reading acquisition is a difficult experiment to conduct. A very recent study from Viersen et al., using longitudinal data from the Dutch Dyslexia Program, carried out this exact experiment, examining whether oral language skills were related to two distinct pathways leading to reading fluency, those pathways being the early pre-literacy pathway of developing phonological awareness, and the late literacy pathway of developing linguistic comprehension (critical for downstream reading comprehension). In addition, they studied the relationship between oral language skills and a history of family risk for dyslexia, in 
order to determine whether one was a covariate of the other in one or both of these literacy pathways (Viersen et al., 2018).

The group found that family risk did in fact have an influence on the acquisition of literacy, in that there were effects observed between family risk and pre-literacy skills, word decoding and reading comprehension. However, despite the family-risk population of children having lower reading performance scores on the neuropsychological tests administered, there was no significant relationship between family risk for the early or late literacy pathways (Viersen et al., 2018). The associations between family risk and these various aspects of literacy were assessed at multiple time points (grade levels) throughout the course of the study for each individual subject, thus accounting for the temporal dimension of when family risk is considered a contributing factor to the onset of dyslexia.

\section{Neuroimaging Studies examining Reading Level and Dyslexia}

In addition to careful consideration of family risk when assessing early precursors of dyslexia, critically-examining the reading level and developmental trajectory of children prior to literacy training is essential in understanding the neurobiology of dyslexia. Structural and functional deficits observed in brain regions/ networks related to reading may not necessarily be specific to dyslexia, but could in fact be due to variability in the process of acquiring literacy (Clark et al., 2014).

In order to parse dyslexia-specific effects from more general reading development effects, an experimental design which separates control subjects into age-matched and reading level-matched groups is necessary. In a 2014 longitudinal study conducted by Dr. Kristi Clark, in conjunction with researchers from Norway, this experimental design was implemented in order to assess whether neuroanatomical changes in the reading network were specific to children diagnosed with dyslexia, or were also observed in reading level-matched control subjects, indicating that these neuroanatomical changes may be the result of a developmental effect on reading. 27 Norwegian children were tested prior to the start of formal literacy training, and after the diagnosis of dyslexia, conveying the full spectrum of literacy acquisition up until a clinical diagnosis was made.

The results illustrated, perhaps unsurprisingly given the plethora of theories related to dyslexia, that the neuroanatomical abnormalities observed prior to acquiring literacy were not in the reading network (perisylvian region of the left hemisphere), but were in fact dispersed throughout the brain in other, indirectly connected networks related to more primary sensory functions, as well as those related to executive functioning (Clark et al., 2014). Abnormalities in the reading network itself were not observed until the children had acquired literacy and received a diagnosis of dyslexia. These results suggest that abnormalities in sensory regions/ networks that are necessary for the optimal functioning of the reading network may be the core cause of dyslexia, rather than disorders of the reading network itself. 


\section{Conclusion}

From this review, it is clear that several theories exist to explain the underlying behavioral, psychological and neurobiological causes of developmental dyslexia. However, the evidence for each of these theories is broadly dispersed across many disciplinary domains, making it increasingly difficult to compare each method and assess whether the relative merits of one may trump the other. More likely than not, considering multiple theories at once, and in a sense following a "hybrid theory" of dyslexia, may provide the most accurate and comprehensive understanding of the underlying, root causes of the disorder. In addition, when attempting to generate clinically-meaningful measures for diagnosing dyslexia prior to literacy acquisition, clinicians and researchers alike should consider multiple other factors related to dyslexia, such as its comorbidity with disorders like ADHD, family risk factors and hereditary information, as well as the reading trajectory of children with dyslexia, and whether or not reading developmental may influence the behavioral and neurological manifestations of dyslexia.

\section{Acknowledgements}

The author would like to acknowledge Drs. Frank Manis, Kristi Clark, Jason Zevin and Mara Mather for their support and mentorship.

\section{Financial Disclosure}

The author has no conflicts of interest, financial or otherwise, to disclose.

\section{References}

1. Bishop, D. V., McDonald, D., Bird, S., \& Hayiou-Thomas, M. E. (2009). Children Who Read Words Accurately Despite Language Impairment: Who Are They and How Do They Do It? Child Development, 80(2), 593-605. doi:10.1111/j.1467-8624.2009.01281.x

2. Boada, R., \& Pennington, B. F. (2006). Deficient implicit phonological representations in children with dyslexia. Journal of Experimental Child Psychology, 95(3), 153-193. doi:10.1016/j.jecp.2006.04.003

3. Bosse, M., Tainturier, M. J., \& Valdois, S. (2007). Developmental dyslexia: The visual attention span deficit hypothesis. Cognition, 104(2), 198-230. doi:10.1016/j.cognition.2006.05.009

4. Buchholz, J., \& Davies, A. A. (2007). Attentional blink deficits observed in dyslexia depend on task demands. Vision Research, 47(10), 1292-1302. doi:10.1016/j.visres.2006.11.028

5. Clark, K. A., Helland, T., Specht, K., Narr, K. L., Manis, F. R., Toga, A. W., \& Hugdahl, K. (2014). Neuroanatomical precursors of dyslexia identified from pre-reading through to age 11. Brain, 137(12), 3136-3141. doi:10.1093/brain/awu229

6. Erbeli, F., Hart, S. A., Wagner, R. K., \& Taylor, J. (2017). Examining the Etiology of Reading Disability as Conceptualized by the Hybrid Model. Scientific Studies of Reading, 22(2), 167-180. doi:10.1080/10888438.2017.1407321

7. Goswami, U., Wang, H. S., Cruz, A., Fosker, T., Mead, N., \& Huss, M. (2011). Language-universal Sensory Deficits in Developmental Dyslexia: English, Spanish, and Chinese. Journal of Cognitive Neuroscience, 23(2), 325-337. doi:10.1162/jocn.2010.21453

8. Morris, R. D., Stuebing, K. K., Fletcher, J. M., Shaywitz, S. E., Lyon, G. R., Shankweiler, D. P., . . . Shaywitz, B. A. (1998). Subtypes of reading disability: Variability around a phonological core. Journal of Educational Psychology, 90(3), 347-373. doi:10.1037//0022-0663.90.3.347

9. Ozernov-Palchik, O., Norton, E. S., Sideridis, G., Beach, S. D., Wolf, M., Gabrieli, J. D., \& Gaab, N. (2016). Longitudinal stability of pre-reading skill profiles of kindergarten children: Implications for early screening and theories of reading. Developmental Science, 20(5). doi:10.1111/desc.12471 
10. Pennington, B. (2006). From single to multiple deficit models of developmental disorders. Cognition, 101(2), 385-413. doi:10.1016/j.cognition.2006.04.008

11. Pennington, B. F., Santerre-Lemmon, L., Rosenberg, J., Macdonald, B., Boada, R., Friend, A., ... Olson, R. K. (2012). Individual prediction of dyslexia by single versus multiple deficit models. Journal of Abnormal Psychology, 121(1), 212-224. doi:10.1037/a0025823

12. Peterson, R. L., \& Pennington, B. F. (2012). Developmental dyslexia. The Lancet, 379(9830), 1997-2007. doi:10.1016/s0140-6736(12)60198-6

13. Ramus, F. (2003). Theories of developmental dyslexia: Insights from a multiple case study of dyslexic adults. Brain, 126(4), 841-865. doi:10.1093/brain/awg076

14. Shaywitz, S. E., \& Shaywitz, B. A. (2005). Dyslexia (Specific Reading Disability). Biological Psychiatry, 57(11), 1301-1309. doi:10.1016/j.biopsych.2005.01.043

15. Viersen, S. V., Bree, E. H., Zee, M., Maassen, B., Leij, A. V., \& Jong, P. F. (2018). Pathways Into Literacy: The Role of Early Oral Language Abilities and Family Risk for Dyslexia. Psychological Science, 29(3), 418-428. doi:10.1177/0956797617736886

16. Wolf, M., \& Bowers, P. G. (1999). The double-deficit hypothesis for the developmental dyslexias. Journal of Educational Psychology, 91(3), 415-438. doi:10.1037//0022-0663.91.3.415 